\title{
The characteristic behavior of TMAH water solution for anisotropic etching on both Silicon substrate and $\mathrm{SiO}_{2}$ layer
}

\author{
Ping-Hei Chen ${ }^{\mathrm{a},{ }^{*}}$, Hsin-Yah Peng ${ }^{\mathrm{a}}$, Chia-Ming Hsieh ${ }^{\mathrm{a}}$, Minking K. Chyu ${ }^{\mathrm{b}}$ \\ ${ }^{a}$ Department of Mechanical Engineering, National Taiwan University, Taipei, Taiwan, ROC \\ ${ }^{\mathrm{b}}$ Department of Mechanical Engineering, University of Pittsburgh, Pittsburgh, PA, USA
}

Accepted 24 March 2001

\begin{abstract}
The present study aims at obtaining the etching rate of $\operatorname{Si}\left(\begin{array}{lll}1 & 0 & 0\end{array}\right)$ surfaces as well as the silicon oxide layer when the surfaces are exposed to tetramethylammonium hydroxide (TMAH) water solution. The influence of TMAH solution concentration and solution temperature on the etching rate is also studied. In this work, the value of TMAH concentration $\left(C_{\mathrm{TMAH}}\right)$ ranges from 2 to $25 \mathrm{wt} \%$ and the etching temperature ranges from 70 to $90^{\circ} \mathrm{C}$. Both n- and p-types silicon substrate were tested. Measured results show that etching changes little with the type of silicon substrate. In addition, the etching rate increases with the solution temperature but decreases with an increase in $C_{\text {TMAH }}$ for $C_{\text {TMAH }}$ greater than $8 \mathrm{wt} . \%$. Among all experimental conditions, a maximum etching rate on the $\mathrm{Si}(100)$ surface reaches a value of $81 \mu \mathrm{m} / \mathrm{h}$ at a solution temperature of $90^{\circ} \mathrm{C}$. Similarly, higher temperature can result in a faster etching rate of the $\mathrm{SiO}_{2}$ surface; while the etching rate of $\mathrm{SiO}_{2}$ surface decreases consistently with an increase in $C_{\mathrm{TMAH}}$.

Measured results of surface roughness $(\mathrm{Ra})$ on silicon substrate are reported after performing a 20 min etching at solution temperatures from 70 to $90^{\circ} \mathrm{C}$. Much smoother substrate surfaces can be observed for higher concentrations. (C) 2001 Elsevier Science B.V. All rights reserved.
\end{abstract}

\section{Introduction}

Anisotropic wet etching is one of the key technologies for microstructure fabrication. It has long been used to make simple structures such as diaphragms and cantilevers for pressure and acceleration sensors. Recently, the demand for fabricating more complicated three-dimensional microstructures, such as ink-jet printing devices and microfluidic systems, is also increasing. In order to improve the quality of these structures, it is essential to collect a complete etching database that contains the etching rates of silicon wafer at various operating conditions. Furthermore, there is also a strong interest in developing processes for fabricating microstructures that are compatible with a commercial complimentary metal-oxide semiconductor (CMOS) process. Precursor chips of microtransducers are required to be integrated with the necessary integrated circuits that are fabricated in a commercial CMOS foundry. Compatibility of the micro-electrical-mechanical-system (MEMS) fabrication

\footnotetext{
*Corresponding author. Tel.: +886-2-2362-1522; fax: +886-2-2363-1755

E-mail address: phchen@ccms.ntu.edu.tw (P.-H. Chen).
}

with a commercial CMOS process allows for monolithic integration with analog and digital circuits, which provides signal conditioning, interface control, and wireless remote communication. After receiving the precursor chips from the CMOS foundry, the microsensors or microactuators are then released by an additional maskless frontside post-processing etching. Examples of these CMOS-compatible microsystems are successfully made as flow sensors [1], temperature sensors [2], infrared thermal displays [3], microwave components $[4,5]$, and solid-state conductometric chemical gas sensors [6].

To manufacture these microtransducers at low cost, there are several requirements on the post-processing etching. Prominently, the chemical etchants must be compatible with materials used in the commercial CMOS processes, namely, silicon oxide, silicon nitride, and exposed aluminum. The etchants cannot contaminate the gate dielectrics with impurities such as mobile alkali ions, which shift the flatband voltage. Such contamination can affect the circuits otherwise. Other requirements for etchants are the ease of handling and safety. At present, the most commonly used etchants can be classified into three types: (1) alkali metal hydroxide [7,8]; (2) diamines-based [9]; (3) quaternary 
ammonium hydroxide [10-12]. Alkali metal hydroxide etchants such as potassium hydroxide water solution $(\mathrm{KOH})$ have high silicon etching rates and anisotropic etching capability. Unfortunately, $\mathrm{KOH}$ is not CMOS compatible because it attacks exposed aluminum and contaminates the gate oxides with mobile alkali metal ions. The diamines-based etchants like ethylenediamine-pyrocatechol (EDP) water with a small amount of pyrazine is a commonly used etchant for the anisotropic etching of single crystal silicon and is extensively applied to silicon micromachining. However, EDP is no longer desirable because it is hazardous and contains long-term toxic effects. When it comes into contact with water solution during cleaning, a visible amber mist develops. Such mist creates a serious inhalation hazard in addition to that caused by the evaporation of the ethylenediamine from the solution. The quaternary ammonium hydroxide group fulfills the requirements of CMOS-compatibility. However, it is still being investigated for its etching characteristics. In this group, tetramethylammonium hydroxide $\left(\left(\mathrm{CH}_{3}\right)_{4} \mathrm{NOH}\right)$ is the preferred etchant because it can achieve a fairly high silicon etching rate. However, despite the many reports in the literature, the etching rate of silicon surface in TMAH solution is still not fully documented, and reproducibility of etching results is still a big problem.

In choosing an etchant, a variety of issues must be considered, for example, ease of handling, toxicity, etching rate, desired topology of the etched bottom surface, ICcompatibility, etching stop, etch selectivity over other materials, mask material and thickness of the mask, etc. Among the aforementioned issues, TMAH has already satisfied some of requirements; like ease of handling, non-toxicity, and IC-compatibility; but the other properties are not exactly understood yet. In this paper, the parameters concerned are the TMAH concentration, solution temperature, and substrate type on the etching rate of both silicon substrate and $\mathrm{SiO}_{2}$ layer. The topology of the etched bottom surface is also one of the subjects studied. The surface roughness of the etched silicon is also measured by conducting a $20 \mathrm{~min}$ etching process.

This paper is presented as follows. The experimental methods and basic information are introduced in Section 2; detailed experimental results and discussions are given in Section 3. In Section 4, conclusions of the present study will be provided.

\section{Experimental apparatus and procedures}

Anisotropic etching experiments were performed on 4 in. (1 $\left.\begin{array}{lll}1 & 0\end{array}\right)$ oriented $n$ - and p-type silicon wafers with a resistance of $1-10 \Omega \mathrm{cm}$. A layer of thermally grown silicon oxide $(5000 \AA)$ served as etching mask material. Etching solutions were prepared by diluting commercially available TMAH 25 wt.\% water solutions, made by J.T. Baker in USA. All experiments were carried out in a glass vessel with a stirrer running at $300 \mathrm{rpm}$ and the temperature of TMAH solutions was kept constant by means of a thermostatically controller. A reflux condenser was used to avoid any change in concentration during the etching process. Before immersing the samples into the TMAH solution, they were dipped in BOE for a few seconds to remove the native silicon oxide and then rinsed in deionized water.

As the etching proceeds, the following three processes are the major factors affecting the etching rate in the system. These processes include reactant transport to the surface (1), surface reaction (2), and reaction product transport away from the surface (3). If either factor (1) or (3) is the dominant factor, the etching rate is limited by diffusion and can be increased by stirring the etchant. If the factor (2) is the major factor; the etching rate is limited by surface reaction-rate and depends strongly on etching temperature, etching material, and etching solution composition. Although the temperature variations will influence both the reaction and diffusion rates, the diffusion-limited processes are relatively insensitive to temperature variations because diffusion-limited processes have lower activation energies than reaction-rate controlled processes. Based on this point, it is believed that adding agitation to the system can effectively reduce the diffusion effects at different concentrations. Besides, agitation can also reduce the accumulation of produced bubbles and reactants. Due to the fact that it is much easier to control etching temperature than stirring rate, a stirrer with constant rotation was used in the etching container for all experiments.

In the present work, three parameters are chosen to vary; namely (1) TMAH solution concentration by weight, (2) etching temperature, and (3) the silicon substrate type; for obtaining the etching characteristics of the $\operatorname{Si}\left(\begin{array}{lll}1 & 0 & 0\end{array}\right)$ surface and that of the passivation silicon dioxide layer. To understand the influence of TMAH solution concentration, a much wider range of TMAH concentration than prior studies was chosen by varying concentration from 2 to $25 \mathrm{wt} . \%$. In the experiments, the etching temperature was selected at three different values, namely 70,80 , and $90^{\circ} \mathrm{C}$. Each experiment at a designated etching condition was conducted three times for checking the repeatability. For the etching process of silicon substrate, the etching temperature was kept at a desired temperature with $\pm 1^{\circ} \mathrm{C}$ accuracy and the etching time was 20 min. Divan et al. [13] reported that the surface roughness for $\mathrm{Si}\left(\begin{array}{lll}1 & 0 & 0\end{array}\right)$ in $\mathrm{KOH}$ etchant could reach a saturated value after a $5 \mathrm{~min}$ etching process. Thus, a 20 min etching time for $\mathrm{Si}\left(\begin{array}{lll}1 & 0 & 0\end{array}\right)$ surface in TMAH solution is considered enough for obtaining the desired surface roughness. However, a much lower etching rate is expected to occur for the passivation silicon dioxide layer than the silicon substrate, and no pre-dipped process is needed. Therefore, the etching time for the silicon dioxide layer was increased to $1 \mathrm{~h}$ to reduce measurement errors.

Etching depth and surface roughness (Ra) of silicon were scanned with a Tensor $\alpha$-step surface profiler with a resolution of $40 \AA$. Afterwards, the etching depths of the passivation silicon dioxide layer were measured by an Ellipsometer 
(Rudolph Research Auto El-III) with a resolution of several angstroms. The characteristics of etched silicon surfaces were also examined by both a scanning electron microscopy (Hitachi S-800) and an optical microscopy (Olympus MX40)

\section{Results and discussions}

\subsection{TMAH concentration}

Fig. 1 shows the etching rates of $n$ - and p-type silicon substrate at three different etching temperatures of 70, 80, and $90^{\circ} \mathrm{C}$ for a wide range of TMAH solution concentration. For the experiments conducted in the present work, the maximum etching rate occurs at a TMAH solution concentration of $8 \mathrm{wt} . \%$ and an etching temperature of $90^{\circ} \mathrm{C}$. The value is $81 \mu \mathrm{m} / \mathrm{h}$. For all three etching temperatures, the etching rate drops with an increase in TMAH concentration for $C_{\mathrm{TMAH}}$ greater than $8 \mathrm{wt}$. $\%$. At lower concentrations, the etching rate varies slightly with the concentration. It can be observed that the type of silicon substrate has little effect on the etching rate of $\operatorname{Si}\left(\begin{array}{lll}1 & 0 & 0\end{array}\right)$ surface within the range of concentration tested in the present work.

Fig. 2 compares the etching rates obtained by Thong et al. [12], Tabata et al. [11], and the present study. The difference in the value of etching rate is not significant for TMAH concentration ranging from 5 to $10 \mathrm{wt} . \%$. A comparison of the maximum etching rates obtained from various studies is shown in Table 1 . Note that in Table 1, the substrate resistance of Tabata et al. [11] is quite different from that of other researches. A much higher substrate resistance, according to the observation by Bhatnagar and Nathan [14], may account for a higher etching rate for Tabata et al. [11] than that of Thong et al. [12]. However, a stirrer was used in the vessel for the present study but not in the study of Thong et al. [12]. It may result in a higher etching rate of the present study than that of Thong et al. [12].

In the present study, the silicon substrate type has little effect on the etching rates for all three temperatures tested. However, the reported results by Thong et al. [12] show that

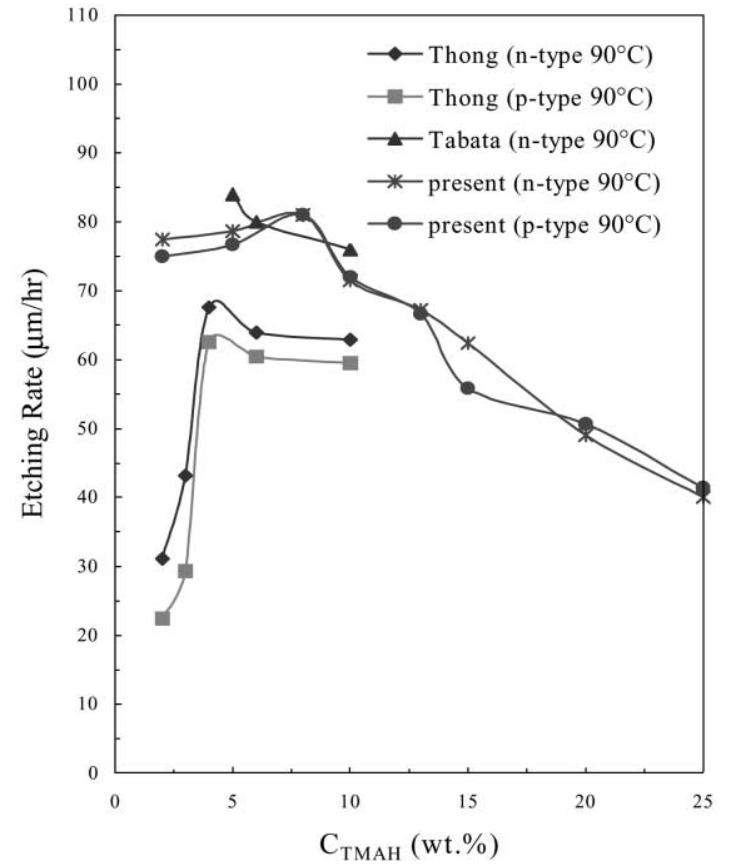

Fig. 2. Comparison of etching rate of $\operatorname{Si}\left(\begin{array}{lll}1 & 0 & 0\end{array}\right)$ surface among various studies.

the etching rate for n-type substrate is always higher than that of p-type. It could be due to the fact that no stirrer was used in their study. Thus, diffusion might have an effect on the etching rate between different types of samples.

\subsection{Characteristics of silicon etched surface}

The silicon surface after the 20 min etching duration was observed by using SEM and OM. A series of optical micrographs were taken to show the surface morphology of the n-type $\operatorname{Si}\left(\begin{array}{lll}1 & 0 & 0\end{array}\right)$ surface at five different TMAH concentrations, as shown in Fig. 3(a)-(e). The test condition for this series of photographs was kept at constant etching temperature of $90^{\circ} \mathrm{C}$ and the TMAH concentration was increased at an interval of 5 wt.\% from Fig. 3(a)-(e). The bright spots in Fig. 3(a)-(c) are pyramidal hillocks found on

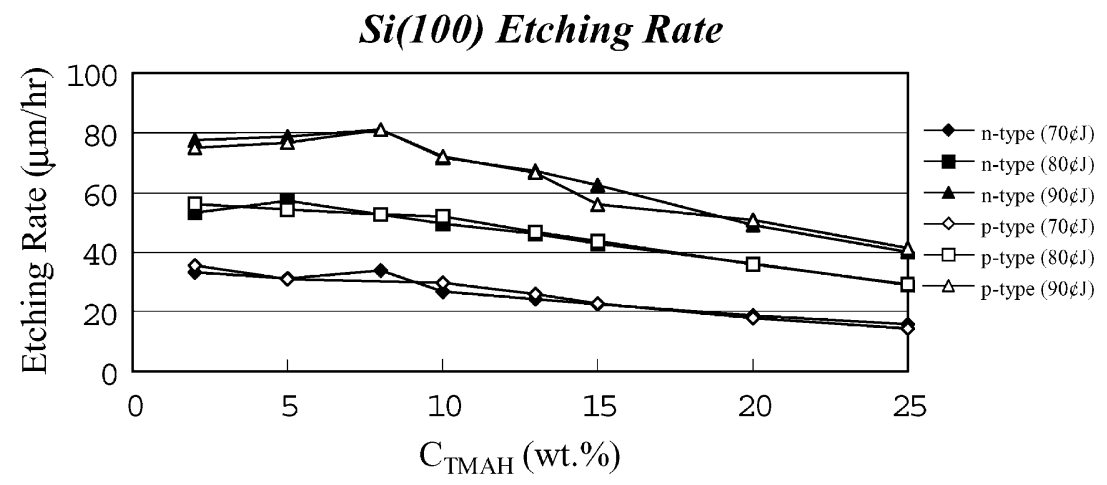

Fig. 1. Effect of TMAH concentration, etching temperature, and type of silicon substrate on the etching rate of $\operatorname{Si}(100)$ surface. 
Table 1

Comparison of the maximum etching rates obtained by Tabata et al. [11], Thong et al. [12], and the present study

\begin{tabular}{llcc}
\hline Parameter & Tabata et al. & Thong et al. & Present result \\
\hline Etchant & TMAH & TMAH & TMAH \\
Concentration (maximum etching rate) $(\%)$ & 5 & 4 & 8 \\
Temperature $\left({ }^{\circ} \mathrm{C}\right)$ & 90 & 90 & $5-10$ \\
Silicon substrate $(\Omega \mathrm{cm})$ & $30-50$ & 70 & $1-10$ \\
Maximum etching rate $(\mu \mathrm{m} / \mathrm{h})$ & 84 & 81 \\
\hline
\end{tabular}

the etched silicon surface. These hillocks with a nearpyramid shape are bounded by $\left\langle\begin{array}{llll}1 & 0 & 1\end{array}\right\rangle$ ledges and near$\left\{\begin{array}{lll}1 & 1 & 1\end{array}\right\}$ planes. However, the hillocks almost disappear on the silicon surface for TMAH concentrations equal to or greater than $20 \mathrm{wt} \%$. Landsberger et al. [15] provided the explanation for the causes for the formation of surface hillocks. It is due to the fact that the anisotropic etching is quite sensitive to the dissolved $\mathrm{Si}$ content. At high dissolved Si content, $\left\{\begin{array}{lll}1 & 0 & 1\end{array}\right\}$ plane is etched much more slowly than $\left\{\begin{array}{lll}1 & 0 & 0\end{array}\right\}$ plane. While the etching process proceeds, solution with a lower TMAH concentration experiences a higher dissolved Si content than that of a solution with a higher TMAH concentration. The large variation in the etching rate of $\left\langle\begin{array}{llll}1 & 0 & 1\end{array}\right\rangle$ ledges and $\left\{\begin{array}{lll}1 & 0 & 0\end{array}\right\}$ planes results

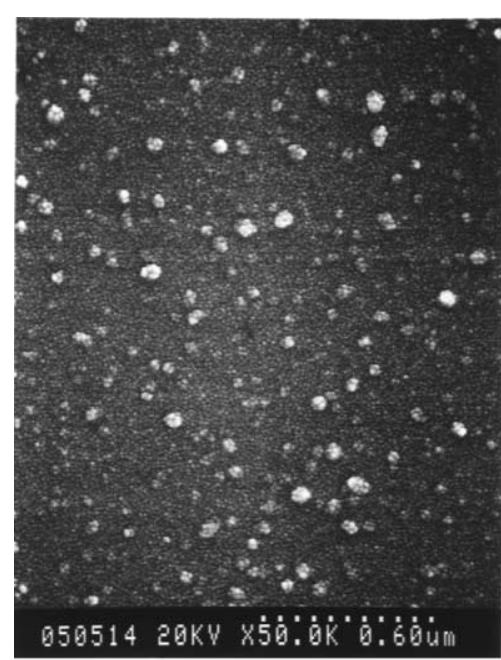

(a)

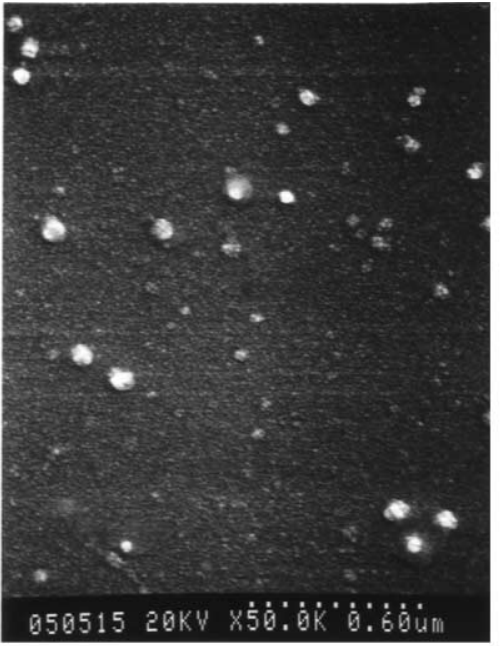

(b)

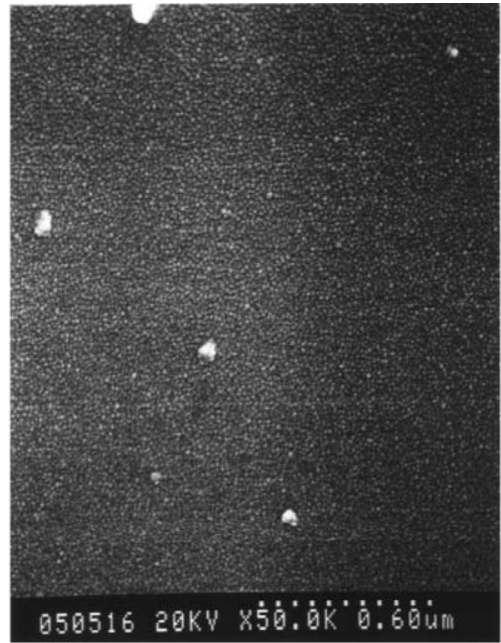

(c)

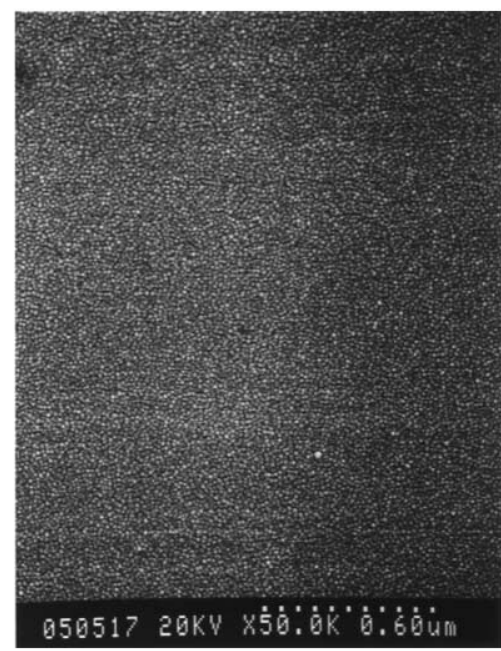

(d)

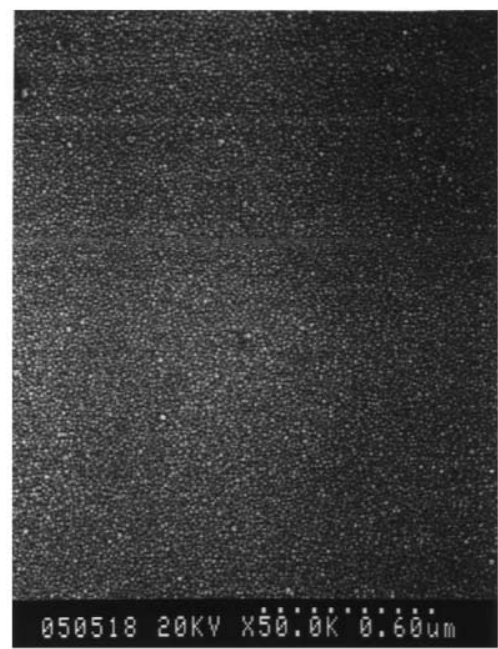

(e)

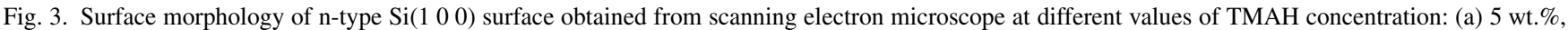
(b) $10 \mathrm{wt} . \%$, (c) $15 \mathrm{wt} \%$, (d) $20 \mathrm{wt} . \%$, and (e) $25 \mathrm{wt} . \%$ at the etching temperature of $90^{\circ} \mathrm{C}$ for $20 \mathrm{~min}$. 


\section{Surface Roughness}

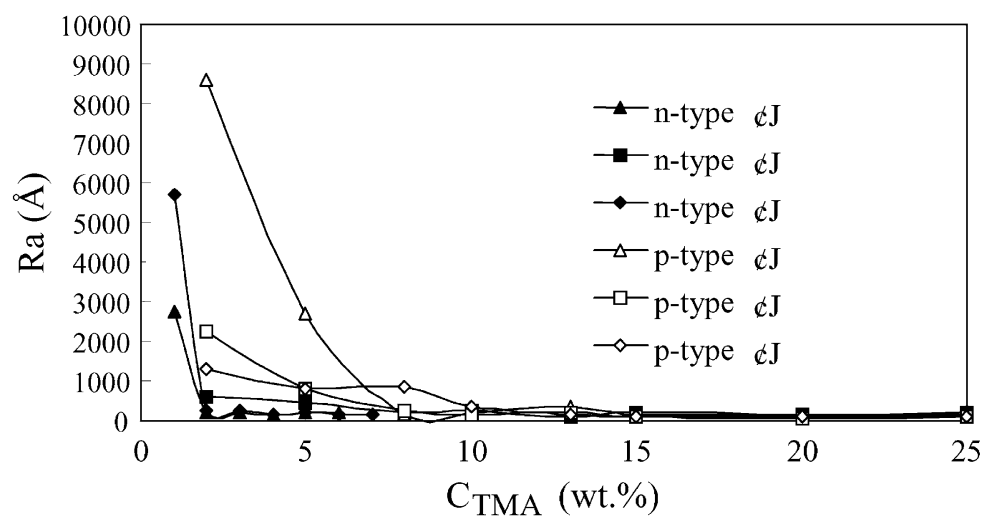

Fig. 4. Effect of TMAH concentration on the surface roughness of $\operatorname{Si}\left(\begin{array}{lll}1 & 0 & 0\end{array}\right)$ surface at the etching temperature of $90^{\circ} \mathrm{C}$ for 20 min.

in the formation of pyramidal hillocks. Similar to our results, Tabata et al. [11] have pointed out that a smooth silicon surface can only be obtained at TMAH concentration higher than $22 \mathrm{wt} . \%$.

The surface morphology shown in the previous optical micrographs can also be confirmed by the surface roughness measurements. As shown in Fig. 4, the surface roughness significantly decreases with an increase in TMAH concentration for both types of silicon substrates. The figure shows the surface roughness in Ra versus TMAH concentration. For TMAH concentration greater than $15 \mathrm{wt} \%$, the surface roughness is less than $200 \AA$ in all test conditions. However, the temperature effect on the surface roughness of etched silicon surface cannot be concluded for the etchant at low TMAH concentrations in the present work.

\subsection{Passivation silicon dioxide layer}

The etching rate of thermal silicon dioxide layer in TMAH solution is roughly 10 times lower than that in $\mathrm{KOH}$. Therefore, it is possible to use $\mathrm{SiO}_{2}$ as a masking layer for a long-time anisotropic etching process. However, the $\left(\begin{array}{lll}1 & 1 & 1\end{array}\right) /\left(\begin{array}{lll}1 & 0 & 0\end{array}\right)$ etching rate ratio of silicon substrate in TMAH solution is a few times higher than that in $\mathrm{KOH}$. Thus, the comparatively low etching rate is the only drawback for TMAH solution when it is implemented as the anisotropic etchant.

The effects of TMAH concentration and temperature on etching rates of the silicon dioxide layer are shown in Figs. 5 and 6 , respectively. The etching rates of the $\mathrm{SiO}_{2}$ layer increases with etching temperature but decreases slightly with TMAH concentration for $C_{\text {TMAH }}$ greater than $5 \mathrm{wt} \%$. For $C_{\text {TMAH }}$ greater than $15 \mathrm{wt} . \%$, the etching rate of $\mathrm{SiO}_{2}$ layer changes little with an increase in TMAH concentration. The etching rate of $\mathrm{SiO}_{2}$ layer seems saturated at a certain threshold concentration of TMAH solution. In addition, as shown in Fig. 5, the etching temperature affects more significantly on the measured etching rates of $\mathrm{SiO}_{2}$ layer in the present study than it did in the study of Thong et al. [12]. It could be due to the fact that no stirrer was used in their study. However, the etching rates of $\mathrm{SiO}_{2}$ layer are in the same order of magnitude between both studies.

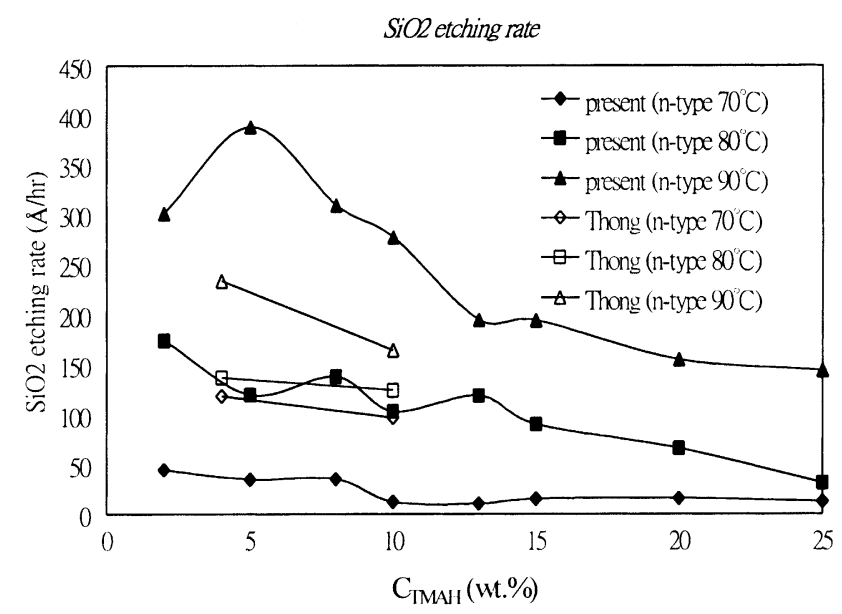

Fig. 5. Etching rate of $\mathrm{SiO}_{2}$ layer at a range of TMAH concentration and at three different etching temperatures.

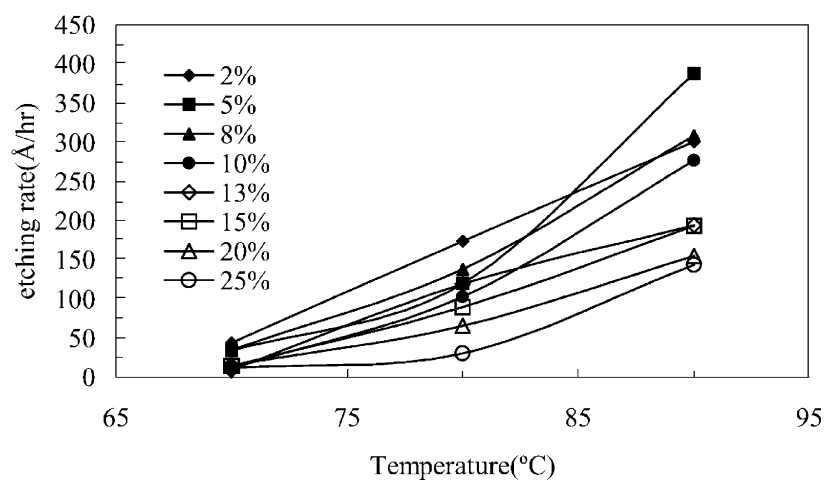

Fig. 6. Effect of etching temperature on the etching rate of $\mathrm{SiO}_{2}$ layer. 


\section{Conclusions}

A systematic study is performed in the present work to investigate the effects of TMAH solution concentrations, etching temperature, and substrate type on the etching rate of both $\mathrm{Si}\left(\begin{array}{lll}1 & 0 & 0\end{array}\right)$ substrate and $\mathrm{SiO}_{2}$ layer. It was found that the maximum etching rate of $\operatorname{Si}(100)$ surface occurs at a TMAH solution concentration of $8 \mathrm{wt} . \%$ and an etching temperature of $90^{\circ} \mathrm{C}$ for all the etching conditions tested. For TMAH solution concentration greater than $8 \mathrm{wt} . \%$, the etching rate decreases with an increase in the TMAH solution concentration for all three etching temperatures tested. The etching rate of $\mathrm{Si}\left(\begin{array}{lll}1 & 0 & 0\end{array}\right)$ surface is significantly increased with the etching temperature. In addition, the etching rate of $\operatorname{Si}\left(\begin{array}{lll}1 & 0 & 0\end{array}\right)$ surface differs a little between $\mathrm{n}$ and p-type silicon samples. The roughness of etched silicon surface after a 20 min etching duration was also measured to check the effect of TMAH solution concentration on the surface roughness. The surface roughness of $\operatorname{Si}\left(\begin{array}{lll}1 & 0 & 0\end{array}\right)$ is slightly changed as the TMAH concentration is greater than 20 wt. \%. The surface roughness is around $200 \AA$. The optical micrographs also confirm that the $\operatorname{Si}\left(\begin{array}{lll}1 & 0 & 0\end{array}\right)$ surfaces are quite smooth without appearance of pyramidal hillocks for a TMAH concentration equal to or greater than $20 \mathrm{wt} . \%$.

Furthermore, the effect of TMAH concentration and etching temperature on the etching rate of silicon dioxide layer is also reported in this study. The trends of the etching rates of silicon oxide layer are similar to that of the silicon wafer for the etching parameters studied in the present work. However, the etching rate of $\mathrm{SiO}_{2}$ layer is about three orders of magnitude lower than that of $\operatorname{Si}\left(\begin{array}{lll}1 & 0 & 0\end{array}\right)$ layer.

\section{References}

[1] D. Moser, R. Lenggenhager, H. Baltes, Silicon gas flow sensor using industrial CMOS and bipolar IC technology, Sens. Actuators A 25-27 (1991) 577-581.
[2] E.H. Klaassen, R.J. Reay, G.T.A. Kovacs, Diode-based thermal r.m.s. converter with on-chip circuitry fabricated using CMOS technology, Sens. Actuators A 52 (1996) 33.

[3] M. Parameswaran, A.M. Robinson, D.L. Blackburn, M. Gaitan, J. Geist, Micromachined thermal radiation emitter from a commercial CMOS process, IEEE Electron Device Lett. 12 (1991) 57.

[4] V. Milanovic, M. Gaitan, E.D. Bowen, M.Z. Zaghloul, Micromachined microwave transmission lines in CMOS technology, IEEE Trans. Microwave Theory Technol. 45 (1997) 630-635.

[5] A. Rofougaran, J.Y.-C. Chang, M. Rofougaran, A.A. Abidi, A $1 \mathrm{GHz}$ CMOS RF front-end IC for a direct-conversion wireless receiver, IEEE J. Solid-State Circuits 31 (1996) 880.

[6] J.S. Suehle, R.E. Cavicchi, M. Gaitan, S. Semancik, Tin oxide gas sensor fabricated using CMOS micro-hotplates and in situ processing, IEEE Electron Device Lett. 14 (1993) 118.

[7] H. Seidel, L. Csepregi, A. Heuberger, H. Baumgartel, Anisotropic etching of crystalline silicon in alkaline solutions, J. Electrochem. Soc. 137 (1991) 3612-3626.

[8] K. Sato, M. Shikida, T. Yamashiro, M. Tsunekawa, S. Ito, Roughness of single-crystal silicon surface etched by $\mathrm{KOH}$ water solution, Sens. Actuators A 73 (1999) 122-130.

[9] A. Reisman, M. Berkenbit, S.A. Chan, F.B. Kaufman, D.C. Green, The controlled etching of silicon in catalysed ethylenediaminepyrocatechol-water solutions, J. Electrochem. Soc. 126 (1979) 14061415.

[10] U. Schnakenberg, W. Benecke, P. Lange, TMAHW etchants for silicon micromachining, Technical Digest, in: Proceedings of the 6th International Conference on Solid-State Sensors and Actuators (Transducers'91), Vols. 24-28, San Francisco, CA, USA, 1991, pp. $815-818$.

[11] O. Tabata, R. Asahi, H. Funabshi, S. Sugiyama, Anisotropic etching of silicon in TMAH solutions, Sen. Actuators A 34 (1992) $51-57$.

[12] J.T.L. Thong, W.K. Choi, C.W. Chong, TMAH etching of silicon and the interaction of etching parameters, Sens. Actuators A 63 (1997) 243-249.

[13] R. Divan, H. Camon, N. Moldovan, M. Dilhan, Limiting roughness in anisotropic etching, in: Proceedings of the IEEE Semiconductor Conference CAS'97, Vol. 2, 1997, pp. 553-556.

[14] Y.K. Bhatnagar, A. Nathan, On pyramidal protrusions in anisotropic etching of $\left\langle\begin{array}{lll}1 & 0 & 0\end{array}\right\rangle$ silicon, Sens. Actuators A 36 (1993) 233-240.

[15] L.M. Landsberger, S. Naseh, M. Kahrizi, M. Paranjape, On hillocks generated during anisotropic etching of Si in TMAH, J. Microelectronmech. Systems 5 (1996) 106-116. 